\title{
UMA ANÁLISE DA PRODUÇÃO ACADÊMICA BRASILEIRA SOBRE O COMPORTAMENTO DO CONSUMIDOR DE ALIMENTO ORGÂNICO ENTRE 1997 A 2011
}

\author{
Danilo de Oliveira Sampaio \\ danilo.sampaio@ufjf.edu.br \\ Universidade Federal de Juiz de Fora - Juiz de Fora, MG / Brasil \\ Marlusa Gosling \\ mg.ufmg@gmail.com \\ Universidade Federal de Minas Gerais - Belo Horizonte, MG / Brasil \\ André Francisco Alcântara Fagundes \\ andrefagundes@fagen.ufu.br \\ Universidade Federal de Minas Gerais - Belo Horizonte, MG / Brasil \\ Caissa Veloso e Sousa \\ caissaveloso@yahoo.com.br \\ Faculdade Novos Horizontes - Belo Horizonte, MG / Brasil
}

Recebido em 14/09/2011

Aprovado em 07/11/2012

Disponibilizado em 01/12/2013

Avaliado pelo sistema double blind review

Revista Eletrônica de Administração

Editor: Luís Felipe Nascimento

ISSN 1413-2311 (versão on-line)

Editada pela Escola de Administração da Universidade Federal do Rio Grande do Sul.

Periodicidade: Quadrimestral

Sistema requerido: Adobe Acrobat Reader.

\section{RESUMO}

O objetivo desta pesquisa foi analisar os trabalhos acadêmicos que tratam sobre o comportamento do consumidor de alimento orgânico publicados nos principais periódicos e eventos científicos brasileiros de Administração entre janeiro de 1997 a março de 2011. Optou-se por um meta-estudo que, segundo Richardson (2007), contribui para o melhor conhecimento do tema analisado além de possibilitar o aperfeiçoamento de estudos futuros referentes ao assunto pesquisado. Foram pesquisados os periódicos classificados como A1, A2, B1 e B2 no Sistema Qualis da Coordenação de Aperfeiçoamento de Pessoal de Nível Superior (CAPES). Ademais, também foram considerados os eventos científicos organizados pela Associação Nacional de Pós-Graduação e Pesquisa em Administração (ANPAD). Para filtrar os artigos analisados optou-se por selecionar os trabalhos que continham a palavra "orgânico" no título, assunto ou palavra-chave. Nesta primeira seleção foram apontados 54 artigos publicados em periódicos e $63 \mathrm{em}$ eventos científicos. Na segunda fase analisaram-se quais desses 117 trabalhos tratavam de comportamento do consumidor de orgânicos, identificando-se nesse último filtro 04 artigos publicados em periódicos e 10 em eventos científicos. A análise dos artigos possibilitou conclusões como: (a) a maioria das publicações 
tanto em periódicos quanto em anais são oriundas de Instituições de Ensino Superior localizadas nas Regiões Sul $(41,38 \%)$ e Sudeste $(34,48 \%)$; (b) com relação aos artigos publicados em periódicos e anais, notou-se que a maioria prefere enquanto metodologia trabalhar com entrevistas em profundidade e análise de conteúdo, contudo, há uma sinalização principalmente nos congressos de que a metodologia survey está demonstrando interesses dos pesquisadores; (c) constatou-se que os artigos adotam preferencialmente a coleta de dados primários e estudos do tipo empírico; (d) quanto ao tipo de pesquisa, destaca-se a exploratór:seguida da descritiva e explicativa. Outras análises mostraram que com relação às teorias e conceitos base apurados, há um bom entendimento da academia quanto à importância de fundamentar a base conceitual, bem como há preocupações metodológicas como apoiar os estudos segundo o tipo de amostra e o objetivo de pesquisa. Notou-se que o ponto mais frágil das publicações está no pouco uso de formulação de hipóteses e pressupostos e falta também nos artigos sugestões de recomendações de pesquisas futuras e contribuições que visassem a aplicação gerencial. Por fim, conclui-se que uma amostra envolvendo outros periódicos além daqueles pesquisados e a realização de um novo estudo a partir do ano de 2011 poderão analisar o incremento da pesquisa sobre o comportamento de consumidor de alimentos orgânicos.

Palavras-chave: comportamento do consumidor; alimento; orgânico; meta-estudo.

\title{
AN ANALYSIS OF THE BRAZILIAN'S ACADEMIC PRODUCTION ABOUT THE ORGANIC PRODUCT CONSUMER BEHAVIOR FROM 1997 TO 2001
}

\begin{abstract}
The purpose of this research was to analyze the academic papers that discourse about the organic product consumer behavior published on the main journals and scientific Administration Brazilian events from January 1997 to March 2011. It was chosen a metastudy which, according to Richardson (2007), contributes for a better knowledge of the analyzed theme, in addition to enable the improvement of future studies related to the surveyed subject. The journals that have been surveyed are classified as A1, A2, B1 and B2 in the Qualis System Coordination Improvement of Higher Personnel Level (CAPES). Moreover, the scientific events organized by the National Association of Post-Graduation and Research in Administration (ANPAD) have been also considered. In order to filter the analyzed articles, it was chosen to select all the papers which contained the word "organic" or in the headline, or in the theme or as a keyword. In this first selection were pointed 54 articles and 63 scientific events. In the second phase were analyzed which ones of these 117 papers talked about the consumer's behavior towards organic food, identifying on the former selection 4 articles published on journals and 10 scientific events. The articles analysis enabled conclusions as: a. most part of the publications as in journals as in proceedings arise from Higher Educational Institutions located on the South $(41,38 \%)$ and Southeast $(34,48 \%)$; b. in relation to the articles published on journals and proceedings it was noticed that the majority prefers as a methodology work with surveys in depth and content analysis, nevertheless there is a sign identified during congress showing that this kind of methodology called survey has been showing the researchers` interest; c. it was found that the articles rather adopt the primary data collection and studies of the empirical kind; $d$. as to the researchers type it was highlighted the exploratory followed by the descriptive and explanatory. Other analysis showed that with respect to the theories and basic concepts established, there is a
\end{abstract}

REAd | Porto Alegre - Edição 76 - N 3 - setembro/dezembro 2013 - p. 620-645 
Uma análise da produção acadêmica brasileira sobre o comportamento do consumidor de alimento orgânico entre 1997 a 2011

good stand-in from the academy as to the importance of substantiate the conceptual basis as well as to support the studies according to the kind of sample or the research's purpose. It was noticed that the weakest part of these publications is the sparse use of formulation of hypothesis and assumptions, missing also in the articles suggested recommendations for future researches and contributions aimed at the management application. Finally, it is concluded that a sample involving other journals in addition to those surveyed and the following study from 2011, it will be possible to analyze the research's increasing about the consumer's behavior towards organic food.

Keywords: consumer’s behavior; food; organic; meta-study.

\section{ANÁLISIS DE LA PRODUCCIÓN ACADÉMICA SOBRE EL COMPORTAMIENTO DEL CONSUMIDOR BRASILEÑO DE ALIMENTOS ORGÁNICOS ENTRE 1997 Y} 2011

\section{RESUMEN}

El propósito de este estudio fue analizar los trabajos académicos que tratan sobre el comportamiento de los consumidores de alimentos orgánicos publicados en las principales revistas científicas y conferencias de Administración de Brasil entre enero de 1997-marzo de 2011. Se realizó un meta-estudio que, de acuerdo con Richardson (2007), contribuye a una mejor comprensión del tema, así como búsquedas en el perfeccionamiento de los futuros estudios sobre el tema. Se realizaron búsquedas en las revistas clasificadas como A1, A2, B1 y B2 en el Sistema Qualis del la Coordinación de Perfeccionamiento de Nivel Superior (CAPES). Además, ha considerado también los eventos científicos organizados por la Asociación Nacional de Estudios de Posgrado e Investigación en Administración (ANPAD). Para filtrar los artículos analizados se decidió seleccionar las obras que contenían la palabra "orgánico" en el título, tema o una palabra clave. En esta primera selección se identificaron 54 artículos publicados en 63 revistas y en reuniones científicas. En la segunda fase se analizaron cuáles de estos 117 documentos han considerado el comportamiento de los consumidores de alimentos orgánicos, destaca de este filtro final 04 artículos publicados en revistas y 10 en reuniones científicas. El análisis de los artículos como las posibles conclusiones indican que: (a) la mayoría de las publicaciones en revistas y en ambos procedimientos son de las instituciones de educación superior ubicado en el Sur $(41,38 \%)$ y Sudeste $(34,48 \%)$; (b) con respecto a los artículos publicados en revistas y actas de congresos, se observó que la mayoría prefiere trabajar en una metodología de entrevistas en profundidad y análisis de contenido. Sin embargo, hay una señal sobre todo en el Congreso que la metodología de la encuesta ha indicado interés para investigadores; (c) se encontró que los artículos aprobados en lugar de la recolección de datos primarios y los estudios empíricos sobre el tipo; (d) el tipo de investigación, está la exploración seguido descriptiva y explicativa. Un análisis posterior mostró que, en relación con las teorías establecidas y los conceptos básicos, hay una buena comprensión de la academia sobre la base de la importancia de los problemas conceptuales y metodológicos es la forma de apoyar los estudios de acuerdo al tipo de muestra y con fines de investigación. Se observó que el punto más débil de las publicaciones es la poca utilidad en la formulación de hipótesis y suposiciones, y también carecen en los artículos las sugerencias y recomendaciones para contribuciones en futuras investigaciones que buscan la aplicación en la gestión empresarial. Finalmente, se concluye que una muestra considerando otras revistas

REAd | Porto Alegre - Edição 76 - N 3 - setembro/dezembro 2013 - p. 620-645 
Danilo de Oliveira Sampaio, Marlusa Gosling, André Francisco Alcântara Fagundes \& Caissa Veloso e Sousa

de reconocimiento y la realización de un nuevo estudio del año 2011 se puede analizar el crecimiento de la investigación sobre el comportamiento del consumidor de alimentos orgánicos.

Palabras clave: comportamiento del consumidor; alimentos; orgánicos; meta-estudio.

\section{INTRODUÇÃO}

O interesse pela pesquisa em alimentos orgânicos mostra-se crescente no Brasil, Ásia, América do Norte e principalmente na Europa (HOPPE et al., 2010; KRISCHKE; TOMIELLO, 2009; VILAS BOAS; SETTE; PIMENTA, 2008). Para o Research Institute of Organic Agriculture (FIBL, 2008), países como Inglaterra, Holanda, Suíça, Áustria, Alemanha, Austrália, Japão, Canadá e Estados Unidos têm desenvolvido relevantes pesquisas sobre consumo de alimento orgânico.

Zuin e Queiroz (2006) consideram o alimento orgânico como um bem agroalimentar especial, constituído pelas seguintes características de produto: alimento saudável; que considera fundamental a preservação do meio ambiente natural; e que envolve relações sociais e econômicas satisfatórias entre os desejos dos consumidores, produtores e trabalhadores rurais.

Observa-se que algumas características do alimento orgânico vão de encontro com princípios da agricultura sustentável, como a proteção dos recursos naturais e a subsistência e autonomia de grupos sociais envolvidos na produção (CONWAY; BARBIER, 1990). Neste ponto de interseção entre a agricultura sustentável e a agricultura orgânica, surge uma oportunidade de se estabelecer um desenvolvimento econômico aliado ao desenvolvimento social, respeitando os fatores ambientais e ecológicos.

O alimento orgânico ainda valoriza (a) o preparo do solo e da rotação de culturas sem uso de insumo químico; (b) quando o produtor é certificado, os empregados no campo devem possuir treinamento segundo normas de certificação de qualidade; (c) os empregadores atendem aos direitos trabalhistas, sendo vetado o trabalho infantil - outra obrigatoriedade para os produtores certificados; (d) o cultivo, a produção, o processo de embalagem e de entrega do alimento orgânico atende normas de certificação de qualidade; (e) as propriedades nutricionais dos alimentos orgânicos são preservadas e não alteradas em laboratório; (f) na produção orgânica tende-se a existência de uma maior interação entre agricultor e consumidor, podendo chegar a desenvolver co-responsabilidades; e (g) existe um crescimento 
Uma análise da produção acadêmica brasileira sobre o comportamento do consumidor de alimento orgânico entre 1997 a 2011

na demanda nacional e internacional por alimentos agroecológicos (CESCHIM; MARCHETTI, 2009; PIMENTA; VILAS BOAS, 2008).

Belik (1994) enquadra o alimento orgânico na classificação de "produto verde" devido aos seus valores funcionais e ao respeito e proteção dispensados ao meio ambiente. Contudo, Chryssochoidis (2000) aponta que há falta de informação por parte dos consumidores a respeito do conceito de alimento orgânico. Para o autor, isto se deve em parte à falta de informação e divulgação por parte de instituições públicas e privadas.

Em recentes pesquisas desenvolvidas no Brasil é possível observar a falta de conhecimento sobre a correta definição do alimento orgânico (CESCHIM; MARCHETTI, 2009). Neste presente artigo optou-se pela definição do MAPA (2011), o qual destaca o alimento orgânico como um produto que tem como base o atendimento dos processos agroecológicos, os quais contemplam o uso racional do solo, água e ar, respeitando as relações sociais e culturais.

Segundo a Federação das Indústrias do Paraná (FIEP, 2011) a GKF - organização especializada em pesquisa de mercado - avaliou o alimento orgânico na cesta de compra dos brasileiros no ano de 2010, chegando à conclusão que os brasileiros estão consumindo mais alimento orgânico, porém de forma mais tímida que os consumidores europeus. A pesquisa ainda conclui que existe um elevado potencial de consumo entre os brasileiros, principalmente os mais jovens, que atualmente não é o maior grupo consumidor desse tipo de produto.

As características do alimento orgânico têm despertado interesse de pesquisas em diferentes áreas do conhecimento: (a) Ciências da saúde, especificamente na Medicina Alternativa e Nutrição (CRINNION, 2010; WILLIAMSON, 2007; ZANOLI; NASPETTI, 2001); (b) Educação e Gestão Ambiental (GOTSCHI et al., 2010; GUTHMAN, 1998); e (c) Agricultura (MEDAETS, 2003; ROE, 2006; SOUZA, 2006; STRINGHETA; MUNIZ, 2003).

$\mathrm{Na}$ área de comportamento do consumidor alguns autores também têm desenvolvido pesquisas buscando compreender as relações de consumo envolvidas na compra de alimentos orgânicos (KRISCHKE; TOMIELLO, 2009; MENEZES; PADULA; CALLEGARO, 2007; VILAS BOAS; BRITO; SETTE, 2006; VILAS BOAS; SETTE; PIMENTA, 2008).

Para Ceschim e Marchetti (2009), atualmente o estudo do comportamento do consumidor de alimentos orgânicos é considerado uma das tendências marcantes nos estudos de marketing. Neste artigo, o alimento orgânico é estudado sob o ponto de vista do comportamento do consumidor.

REAd | Porto Alegre - Edição 76 - N 3 - setembro/dezembro 2013 - p. 620-645 
Danilo de Oliveira Sampaio, Marlusa Gosling, André Francisco Alcântara Fagundes \& Caissa Veloso e Sousa

A presente pesquisa pretende responder ao seguinte problema: quais são os tipos de estudos relacionados ao comportamento do consumidor de alimento orgânico que estão sendo desenvolvidos na academia brasileira? Respondendo à pergunta de pesquisa, o objetivo geral desse artigo é analisar os trabalhos acadêmicos de comportamento do consumidor de alimento orgânico publicados no Brasil de 1997 a março de 2011. Acredita-se que a análise deste período, que engloba quinze anos, viabilizou a comprovação do crescimento do número de pesquisas a respeito do assunto investigado.

$\mathrm{O}$ artigo, além desta introdução, apresenta em sua estrutura o referencial teórico, que será abordado na sequência, além dos aspectos metodológicos, análise dos dados coletados referentes às pesquisas identificadas e as considerações finais.

\section{REFERENCIAL TEÓRICO}

O referencial teórico desta pesquisa trata primeiramente da importância do comportamento do consumidor enquanto escola teórica de marketing. A discussão acerca dessa escola teórica se faz presente, uma vez que importantes estudos sobre comportamento de compra do alimento orgânico, se considerados os aspectos das ciências sociais, têm como fundamentação seus pressupostos, especialmente, a partir da utilização da Teoria do Comportamento Planejado (TCP) no desenho de modelos preditores desse comportamento. Posteriormente são apresentadas algumas pesquisas que estudaram o comportamento do consumidor de alimento orgânico, tanto no país quanto no exterior.

\subsection{0 comportamento do consumidor}

O estudo do comportamento do consumidor constitui um campo multidisciplinar que despontou interesse após a Segunda Guerra Mundial, envolvendo as áreas da Psicologia, Antropologia e Sociologia. Sua origem se identifica com a teoria econômica, mais especificamente com a microeconomia, para a qual o consumidor é dotado de uma renda limitada, que deve ser alocada entre os bens e serviços de forma a maximizar seu bem estar.

Se considerado o campo de pesquisas na Administração, conforme escolha dos autores para delimitação do escopo do trabalho, a escola do comportamento do consumidor é uma das principais escolas da área de marketing, conforme apontam Sheth, Gardner e Garret (1988). Esta escola responde os motivos pelos quais os mercados se comportam tendo como foco 
Uma análise da produção acadêmica brasileira sobre o comportamento do consumidor de alimento orgânico entre 1997 a 2011

principal o estudo dos consumidores, sendo fundamental para estabelecer um melhor relacionamento entre as organizações e o consumidor.

Dois objetivos se destacam na pesquisa do comportamento do consumidor: a previsão e entendimento deste comportamento e o consequente descobrimento das relações de causa e efeito que possam influenciar ou educar o consumo (ENGEL; BLACKWELL; MINIARD, 2000), o que justifica sua discussão ao se tratar do consumo do alimento orgânico.

Peter e Olson (2009) citam que a pesquisa do comportamento do consumidor é baseada em três grupos principais: (1) organizações que usam o marketing no uso de estratégias mercadológicas; (2) organizações governamentais na adoção de políticas públicas; e (3) os consumidores e compradores organizacionais que baseiam suas atividades em trocas que explicam seu próprio comportamento.

A partir da década de 1960, várias academias se interessaram pelo estudo do comportamento do consumidor, com isso vários modelos comportamentais foram desenvolvidos. Organizações públicas e privadas passaram a utilizar as pesquisas de mercado para monitorar a demanda e selecionar as estratégias de acordo com as tendências de consumo. (SHETH; GARDNER; GARRET, 1988)

Holbrook (1987, p. 131), ao discutir qual o significado ontológico da pesquisa do consumidor, chegou à seguinte definição: “o estudo dos processos onde há aquisição, uso e disposição de todos os tipos de produtos que têm valor para aquilo que o homem deseja". O autor comenta que a vida é um contínuo consumo e que envolve atividades que visam objetivo, satisfação de necessidades e desejos. Dessa forma, o comportamento do consumidor não deve ser visto apenas como uma ação racional dos compradores, mas também como uma atividade relacionada com emoções, fantasias e sentimentos, inclusive os relacionados às experiências de consumo anteriores. O autor ainda explica que o simbolismo possui significante ligação com o consumo, pois os indivíduos compram bens não apenas porque precisam destes, mas porque desejam mostrá-los como forma de pertencer a um grupo social, ou mesmo para expressar suas personalidades.

Autores como Peter e Olson (2009) destacam o comportamento do consumidor como uma das disciplinas mais importantes do marketing. Hawkins, Mothersbaugh e Best (2007) ampliam a definição de comportamento do consumidor para além das trocas entre comprador e vendedor. Alguns autores consideram o comportamento do consumidor como área estratégica, como Blackwell, Miniard e Engel (2005). Os autores sugerem que as 
Danilo de Oliveira Sampaio, Marlusa Gosling, André Francisco Alcântara Fagundes \& Caissa Veloso e Sousa

organizações devem exercitar o estudo permanente do mercado e observar as atitudes dos seus compradores.

Solomom (2002), apresentando uma visão mais psicológica sobre o comportamento do consumidor, defende os consumidores como atores no palco do mercado, possuindo papéis diferentes dependendo das circunstâncias ambientais. Para o autor, esse comportamento é um processo contínuo que envolve questões de pré-compra, compra e pós-compra, além de funções diferenciadas que podem ser exercidas por um ou mais indivíduos, como: influenciador, comprador, usuário e avaliador.

Já de acordo com Bagozzi, Gürhan-Canli e Priester (2002), o comportamento do consumidor é baseado em trocas e benefícios mútuos, envolvendo inclusive aspectos simbólicos fundamentais para os consumidores. Os autores destacam as atitudes e intenções comportamentais, pois, para eles, estes aspectos estão diretamente ligados à intenção de compra.

Sheth, Gardner e Garret (1988) propuseram a divisão do marketing enquanto ciência em escolas, de acordo com quatro perspectivas fundamentais. As perspectivas relacionam o fator "econômico" com o fator "interatividade".

A escola do comportamento do consumidor é visto na perspectiva não-econômica e não-interativa (Figura 1).

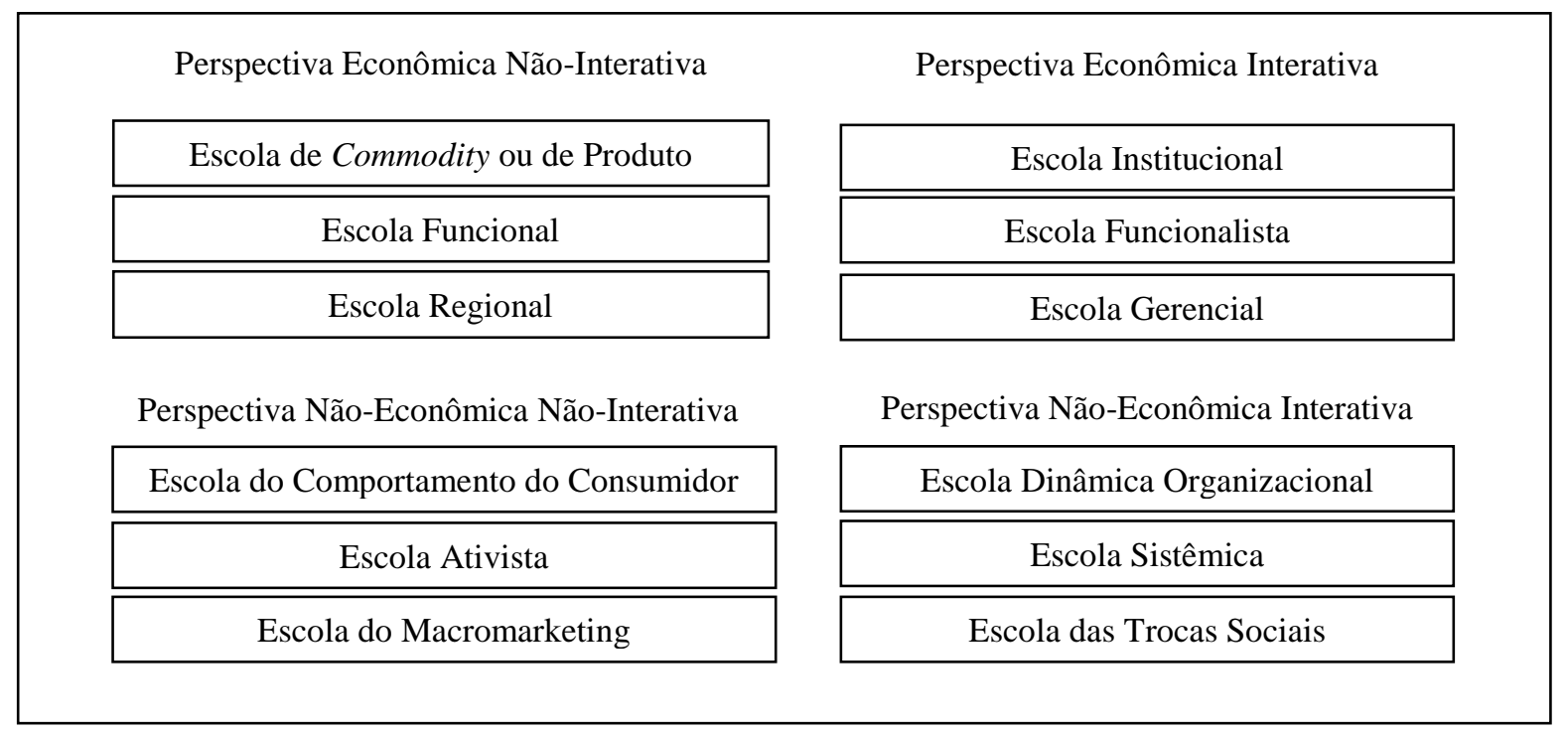

Figura 1 - Escolas teóricas do marketing

Fonte: adaptado de Sheth, Gardner e Garret (1988) 
Uma análise da produção acadêmica brasileira sobre o comportamento do consumidor de alimento orgânico entre 1997 a 2011

\subsection{O comportamento do consumidor de alimento orgânico}

As contribuições de autores estrangeiros têm sido marcantes com relação ao tema de comportamento do consumidor de alimento orgânico. Neste sentido, é interessante discutir a seguir algumas destas contribuições.

Tarkiainen e Sundqvist (2005) pesquisaram o comportamento de consumidores de alimentos orgânicos (pão e farinha orgânica) na Finlândia. Os autores aplicaram a técnica de modelagem por equações estruturais para entender a relação entre as normas subjetivas, atitudes e intenções de compra do consumidor de alimentos orgânicos. O modelo proposto pelos autores mostrou-se eficaz quanto apontou que a decisão do consumidor em comprar orgânicos se baseia no bem estar e na questão ambiental. A relação entre as atitudes para a compra de alimentos orgânicos e a intenção de comprar alimento saudável foi positiva e significativa.

Tarkiainen e Sundqvist (2005) concluíram que as atitudes e a disponibilidade influenciam significativamente na intenção de consumo de tomates orgânicos. Os autores concluíram que os construtos incerteza percebida e norma subjetiva não apresentaram efeito significativo na intenção de compra, e que em relação às normas subjetivas, ou seja, a pressão social para exercer determinado comportamento, as variáveis "respeito" e "admiração" por quem utiliza o alimento orgânico, não são elementos significantes na determinação da intenção de consumo de tomates orgânicos.

Arvola et al. (2008) estudaram as atitudes morais e afetivas por meio do modelo da Teoria do Comportamento Planejado (TCP). Os autores buscaram verificar que fatores poderiam predizer as intenções de compra de produtos orgânicos. A pesquisa foi aplicada em três países da Europa e os resultados apontaram parcialmente que o uso de medidas morais e afetivas interfere na intenção de compra do consumidor de alimento orgânico.

Segundo os autores a decisão de consumo de alimentos orgânicos pode ser tomada tanto por valores abstratos como por atributos deste tipo de alimento. Por valores abstratos, Arvola et al. (2008) apontam como sendo o hedonismo, benevolência, universalismo, estimulação, auto-suficiência e conformidade. Ainda segundo a pesquisa, as atitudes morais e afetivas correspondem às crenças de controle que envolve a TCP.

Dean, Raats e Shepherd (2008) também usaram a TCP para examinar o impacto dos interesses morais na intenção de compra de dois alimentos distintos: maças e pizza orgânica. A conclusão apontou que nos dois produtos o moral positivo influencia significantemente a REAd | Porto Alegre - Edição 76 - N 3 - setembro/dezembro 2013 - p. 620-645 
Danilo de Oliveira Sampaio, Marlusa Gosling, André Francisco Alcântara Fagundes \& Caissa Veloso e Sousa

predição da intenção de compra. Os autores verificaram também que a atitude afetiva é um forte indicador da intenção de compra tanto para a maça quanto para a pizza orgânica.

Outra pesquisa que relata o comportamento do consumidor de alimento orgânico foi desenvolvida por Shepherd, Magnusson e Sjödén (2005), que realizaram uma survey na Suécia com 2000 respondentes entre 18 e 65 anos de idade. Os alimentos orgânicos escolhidos neste trabalho para participarem do estudo foram leite, carne, batata e pão, por serem produtos importantes na dieta dos suecos.

Os autores questionaram na pesquisa os critérios de compra dos consumidores e as crenças que tinham a respeito dos alimentos orgânicos. Os resultados sugerem que apesar dos consumidores revelaram atitudes positivas em relação ao alimento orgânico, não foram encontradas evidências suficientes que esta atitude positiva interfira diretamente na compra desses produtos. Apesar dos entrevistados afirmarem que o alimento orgânico é saudável, eles consideram que o preço de venda deveria ser o mesmo dos alimentos convencionais. Ademais, os consumidores apontaram que o sabor do alimento orgânico não foi um diferencial na sua escolha, contudo eles acreditam que esse produto preserva o meio ambiente por não usar insumos químicos e poluentes.

\section{ASPECTOS METODOLÓGICOS}

Este estudo sobre análise da produção acadêmica brasileira do comportamento do consumidor de alimentos orgânicos apresenta caráter descritivo, levando-se em consideração a busca, classificação e análise de artigos científicos em periódicos e congressos nacionais (RICHARDSON, 2007).

Estudos referentes à análise do estado da arte da publicação brasileira na área de marketing têm sido desenvolvidos esporadicamente, conforme visto nos trabalhos de Fagundes et al. (2010), Perin et al. (2000) e Vieira (2001). Esse tipo de pesquisa busca colaborar para a discussão e desenvolvimento de novos estudos na área considerada (FLICK, 2004).

Para a coleta de dados, optou-se por analisar os trabalhos das seguintes fontes: (i) artigos publicados em eventos científicos da Associação Nacional de Pós-Graduação e Pesquisa em Administração (ANPAD), totalizando nove eventos; e (ii) periódicos nacionais classificados com os estratos A1, A2, B1 e B2 - na área de Administração, Ciências Contábeis e Turismo - segundo o sistema Qualis da Coordenação de Aperfeiçoamento de Pessoal de Nível Superior (CAPES), que englobou 113 periódicos.

REAd | Porto Alegre - Edição 76 - N 3 - setembro/dezembro 2013 - p. 620-645 
Uma análise da produção acadêmica brasileira sobre o comportamento do consumidor de alimento orgânico entre 1997 a 2011

Ressalta-se que dada a necessidade de delimitar o escopo do trabalho, optou-se por concentrar a análise na área de Administração e suas correlatas, segundo classificação da CAPES, contudo, entende-se que o assunto "alimento orgânico", compreenda um campo de estudo em distintas áreas do conhecimento, como a Sociologia, Antropologia, Comunicação, Agronomia, Engenharia Genética, Engenharia Agrícola, Economia e a Psicologia.

Os filtros usados para se selecionar os artigos a serem analisados foram: título do artigo, o assunto e as palavras-chave. Nestes filtros a palavra pesquisada foi o termo "orgânico". Com isso, foram selecionados 54 artigos publicados em periódicos e 63 em eventos científicos. Após uma segunda análise que englobou uma verificação individual dos trabalhos para se identificar e selecionar aqueles que tratavam do estudo do comportamento do consumidor de alimentos orgânicos, foi possível apontar os trabalhos que realmente fariam parte do estudo, os quais totalizaram 14 artigos, sendo 04 publicados em periódicos e os demais em eventos científicos.

Um formulário contendo 14 questões foi utilizado na análise dos artigos selecionados. Estas 14 variáveis, as quais são explicadas a seguir, foram adaptadas de Botelho e Macera (2001); Perin et al. (2000); Pinto e Lara (2007); e Vieira (2001). Ademais, foram inseridas outras que não foram contempladas nesses estudos. Após essa avaliação, os dados coletados foram tabulados e analisados utilizando-se os softwares Excel 2007 e SPSS 15.0. Posteriormente procedeu-se a elaboração das considerações finais e recomendações dos autores.

As variáveis consideradas foram:

a) Dados do artigo: local de publicação, ano de publicação e quantidade de autores.

b) Dados dos autores: gênero, titulação, instituição a qual o autor está vinculado, e o Estado da instituição.

c) Tipo de estudo: empírico ou teórico.

d) Abordagem da pesquisa: qualitativa ou quantitativa.

e) Natureza da pesquisa: exploratória, descritiva, causal ou outra.

f) Metodologia de pesquisa: aplicação de questionários estruturados, experimentos, estudo de caso, etnografia, grupo focal, entrevistas em profundidade, pesquisa bibliográfica ou outra.

g) Tipos de dados coletados: primário ou secundário.

h) Abrangência do estudo: longitudinal ou transversal.

i) Instrumentos de coleta de dados utilizados. 
Danilo de Oliveira Sampaio, Marlusa Gosling, André Francisco Alcântara Fagundes \& Caissa Veloso e Sousa

j) Características técnicas da pesquisa: teorias e conceitos de base; objetivos da pesquisa; questões de pesquisa; hipóteses ou pressupostos de base; tipos de dados coletados; forma de coleta de dados; tipo de amostra; limites do estudo; recomendações para pesquisas futuras; e recomendações para aplicação prática.

k) Tipo de alimento orgânico encontrado na pesquisa.

1) Tipo de agricultura citada na pesquisa.

m) Questões de qualidade e de meio ambiente encontrados na pesquisa.

n) Questões referentes ao trabalhador rural encontrados na pesquisa.

A seguir tem-se a análise dos artigos de comportamento do consumidor de alimento orgânico segundo critérios metodológicos apontados anteriormente, entre o período de 1997 a março de 2011. Aqui cabe salientar que o período foi delimitado pelos autores tendo em vista a consulta a materiais anteriores a 1997, a qual ficou prejudicada, uma vez que algumas fontes não disponibilizaram artigos anteriores a essa data.

\section{ANÁlise dos ARTigos de COMPORTAMENTO DO CONSUMIDOR DE ALIMENTO ORGÂNICO NO BRASIL ENTRE O PERÍODO DE 1997 A MARÇO DE 2011}

A análise dos resultados foi dividida em duas partes. Inicialmente, optou-se por apresentar algumas das pesquisas dos autores brasileiros sobre a temática que foi objeto de estudo nesse trabalho. A segunda etapa consiste na análise dos artigos, que foram selecionados nas fontes acadêmicas anteriormente citadas, e que tratavam do comportamento dos consumidores de alimentos orgânicos. Para essa última etapa desenvolve-se uma análise estatística dos artigos elegidos para o estudo.

\subsection{Pesquisas de autores brasileiros sobre o comportamento do consumidor de alimento orgânico}

Os trabalhos aqui destacados, mesmo sendo em pequeno número, possuem méritos devido ao pioneirismo na pesquisa ligado ao tema e ao apontamento de dados empíricos que fornecem informações relevantes sobre o comportamento do consumidor brasileiro de alimento orgânico. A maioria das pesquisas ocorreu no sul do Brasil e algumas no sudeste.

Krischke e Tomiello (2009) objetivaram em sua pesquisa identificar as motivações das escolhas dos consumidores por alimentos orgânicos. Os autores realizaram uma pesquisa REAd | Porto Alegre - Edição 76 - N 3 - setembro/dezembro 2013 - p. 620-645 
Uma análise da produção acadêmica brasileira sobre o comportamento do consumidor de alimento orgânico entre 1997 a 2011

qualitativa em um supermercado de Florianópolis-SC e chegaram à conclusão de que os principais motivos que influenciaram os consumidores a comprarem alimentos orgânicos foram: (1) vida saudável (saúde devido a uma dieta rica em alimentação orgânica); (2) qualidade de vida; (3) confiança na marca e (4) qualidade do produto. Os resultados dos trabalhos dos autores apresentam semelhanças em outras pesquisas desenvolvidas no Brasil (ARCHANJO; BRITO; SAUERBECK, 2001; CESCHIM; MARCHETTI, 2009; CUPERSCHMID; TAVARES, 2002; RUCINSKI; BRANDENBURG，2002; SLUZZS; PADILHA; MATTOS, 2008).

Krischke e Tomiello (2009) observaram um fato importante na sua pesquisa: que o alimento orgânico está inserido em uma rede de símbolos ecológicos na qual a alimentação faz parte. Este fato ligando o simbolismo ecológico ao alimento orgânico também foi verificado por Archanjo, Brito e Sauerbeck (2001). As autoras buscaram compreender qual o valor simbólico atribuído ao alimento orgânico por parte dos consumidores curitibanos, e concluíram que a produção e o consumo desse tipo de produto fazem parte de um movimento que propõem mudanças além do comportamento alimentar, ou seja, envolve também uma atitude forte em relação à preservação do meio ambiente.

Conforme sinaliza em outra pesquisa nacional, o alimento orgânico possui apelo positivo em relação às questões ambientais segundo Sluzzs, Padilha e Mattos (2008). Ademais, as autoras - que desenvolveram um estudo de caso na organização Namastê, fabricante de chás orgânicos - concluíram que a agregação de valor aos produtos orgânicos via inovação e pesquisa, possibilitou uma redução nos custos de produção, melhorou a qualidade de vida dos produtores e aumentou a competitividade da organização.

Rucinski e Brandenburg (2002), Archanjo, Brito e Sauerbeck (2001) e Ceschim e Marchetti (2009) desenvolveram pesquisas estudando o perfil dos consumidores de alimentos orgânicos na capital paranaense. Sendo que ambos também confirmaram que o consumidor de alimento orgânico valoriza o meio ambiente e o fator ecológico.

Ceschim e Marchetti (2009) aplicaram uma pesquisa qualitativa por meio de entrevistas em profundidade na região Metropolitana de Curitiba. Os autores entrevistaram consumidores de orgânicos entre 18 a 70 anos de idade pertencentes às classes socioeconômicas A1, A2, B1 e B2 segundo o Critério Brasil. Ceschim e Marchetti (2009) verificaram que os consumidores de alimentos orgânicos se autoavaliam como inovadores, possuindo imagem de pessoas que valorizam atividades esportivas, a natureza e a qualidade de vida. Outro dado na pesquisa dos autores aponta que os consumidores de orgânicos 
Danilo de Oliveira Sampaio, Marlusa Gosling, André Francisco Alcântara Fagundes \& Caissa Veloso e Sousa

percebem as vantagens do alimento orgânico, e estão dispostos a pagar mais por estes produtos, porém, citam dificuldade em relação à experimentação e observação dos orgânicos nos seus locais de venda.

Zamberlan, Büttenbender e Sparemberger (2002) também afirmam que a questão do meio ambiente é considerada como positiva influenciadora pelos consumidores de alimentos orgânicos. Os autores desenvolveram uma survey com 144 consumidores em um pequeno município no noroeste do Rio Grande do Sul, e concluíram que as seguintes variáveis refletem os principais motivos que levam os consumidores a adquirirem o alimento orgânico: (i) saúde; (ii) segurança do alimento; e a (iii) redução de problemas ambientais. Zamberlan, Büttenbender e Sparemberger (2002) salientam ainda que o consumidor de alimento orgânico é exigente e valoriza a oferta de produtos seguros.

Com relação à variável preço, Cuperschmid e Tavares (2002); Pimenta et al. (2009); Rucinski e Brandenburg (2002); Zamberlan, Büttenbender e Sparemberger (2002) verificaram nas suas pesquisas que os consumidores de alimento orgânico estão dispostos a pagar um sobrepreço para terem em suas mesas um alimento que consideram ter melhor qualidade. Corroborando com esses estudos, para Coltro (2006) o consumidor de alimento orgânico reconhece o valor agregado deste produto, e está disposto a pagar a mais para conquistar uma saúde melhor. Ainda considerando a questão da precificação, observa-se que este tipo de consumidor possui poder aquisitivo acima da média brasileira, pois $45,1 \%$ dos consumidores de alimento orgânico têm renda acima de doze salários mínimos ao mês (KRISCHKE; TOMIELLO, 2009).

Outro atributo de destaque em estudos sobre o alimento orgânico é a saúde. Segundo Krischke e Tomiello (2009) o consumidor desse tipo de produto tende a ser exigente em termos de qualidade e consciente em relação aos danos e aos riscos que os agrotóxicos utilizados nos alimentos tradicionais causam à saúde humana. Ainda nesse aspecto, Pimenta et al. (2009) apontam que $33 \%$ dos consumidores de alimentos orgânicos se dizem compradores frequentes, e que $82,6 \%$ afirmam que o alimento tradicional possui agrotóxico e fazem mal à saúde, enquanto que a opção pelo alimento orgânico é mais saudável.

A variável saúde é encontrada em outros estudos sobre o comportamento do consumidor de alimento orgânico. O estudo empreendido por Vilas Boas, Sette e Brito (2006), que utilizou a metodologia de escalonamento de valores - ou laddering - em feiras livres na cidade de Belo Horizonte-MG, sugere que os consumidores de orgânicos prezam pelo cuidado com a saúde pessoal e familiar, sendo orientados por valores como longevidade, 
Uma análise da produção acadêmica brasileira sobre o comportamento do consumidor de alimento orgânico entre 1997 a 2011

tranquilidade e felicidade. Menezes, Padula e Callegaro (2007), que desenvolveram uma pesquisa sobre vinhos orgânicos, também concluíram que, na opinião do consumidor, o sabor, a cor e a textura desse produto estão relacionados a uma melhor saúde.

Ainda considerando a questão da percepção de valores por parte dos consumidores, Pimenta e Vilas Boas (2008) identificaram na cidade de Uberlândia-MG evidências que confirmam a demanda dos consumidores por frutas, legumes e verduras orgânicas segundo a estrutura de atributos, consequências e valores para atender a teoria de meios e fins.

Em se tratando de valores e estilo de vida dos consumidores de orgânicos é interessante citar alguns estudos. Krischke e Tomiello (2009) observaram a compra por indução dos alimentos orgânicos, pois os autores acreditam que a compra consciente depende da melhoria do grau de conhecimento a respeito do alimento orgânico. Ao analisar o estilo de vida do consumidor de alimento orgânico os autores chegaram a uma constatação: este consumidor valoriza o meio ambiente, é consciente dos seus atos e valoriza a saúde.

Nesse sentido, cabe citar Pimenta e Vilas Boas (2008), que concluíram que o estilo de vida do consumidor de alimento orgânico é próximo do conceito ego-trip conforme citam Guillon e Willequet (2003) - estilo que valoriza autonomia, prazer, bem-estar, vida saudável e relação harmoniosa com a natureza. Fato semelhante foi observado por Krischke e Tomiello (2009), quando os respondentes destacaram que o alimento orgânico não agride a natureza, contudo, associavam esta afirmação à saúde, e não ao meio ambiente, sendo este tema pouco citado pelos entrevistados.

Krischke e Tomiello (2009) apontam que o consumidor de orgânicos normalmente é consciente em relação aos riscos que os agrotóxicos causam à saúde humana, ao mesmo tempo em que percebem que o alimento orgânico como possível opção para prevenir diferentes doenças.

A pesquisa de Pimenta e Vilas Boas (2008) encontrou os seguintes valores com relação ao consumidor de alimento orgânico: valor de bem estar; felicidade; nostalgia; qualidade de vida; tempo para fazer outras coisas; e valorização da humanidade e viver mais e melhor. Os autores também revelam algumas das preocupações dos consumidores em relação aos alimentos, como: ausência de insumos químicos; características sensoriais de sabor, cheiro e cor; e buscar por um alimento de melhor qualidade, mais concentrado, nutritivo e de menor perecibilidade do que os alimentos da agricultura tradicional.

Ainda com relação ao perfil do consumidor de alimento orgânico, Krischke e Tomiello (2009) apresentaram o depoimento de vinte consumidores em relação à pergunta: O que o(a)

REAd | Porto Alegre - Edição 76 - N 3 - setembro/dezembro 2013 - p. 620-645 
Danilo de Oliveira Sampaio, Marlusa Gosling, André Francisco Alcântara Fagundes \& Caissa Veloso e Sousa

leva a escolher os alimentos orgânicos? Os autores caracterizaram o perfil de cada respondente e as narrativas no que denominaram síntese da pesquisa, e concluíram que as principais razões de consumo desse tipo de alimento em Florianópolis foram possuir uma vida saudável e uma melhor qualidade de vida. Os autores relatam sobre o perfil dos consumidores de orgânicos: 34\% têm entre 31 a 40 anos de idade; as mulheres são as que mais procuram pelo alimento orgânico (66\%); e 58\% dos consumidores possuem terceiro grau completo.

Hoppe et al. (2010) realizaram uma survey com 446 consumidores de tomates orgânicos em um supermercado e uma feira ecológica na cidade Porto Alegre, RS. Os autores aplicaram a Teoria do Comportamento Planejado (TCP) de Ajzen e Fishbein (2005) com o objetivo de compreender melhor como os consumidores planejam suas compras. Hoppe et al. (2010) apontam nos resultados que as atitudes e a disponibilidade influenciam significativamente na intenção de consumo do alimento orgânico. Destaca-se que no estudo desses autores os construtos norma subjetiva e incerteza percebida não apresentaram efeito significativo na intenção de compra de tomates orgânicos.

Observando os relatos até então apontados em relação ao perfil do consumidor brasileiro de alimento orgânico, verifica-se que este consumidor é bem instruído, exigente, está numa fase da vida considerada adulta e se encontra, na maioria das vezes, entre as classes sociais A1, A2, B1, B2, segundo o critério Brasil. Em relação aos valores os consumidores de orgânicos prezam por uma vida saudável, se preocupam com o meio ambiente e a questão ecológica, e se mostram interessados pelo bem-estar próprio e dos outros, apresentando atitudes positivas perante a compra de alimentos orgânicos.

A seguir são analisados os artigos de comportamento do consumidor de alimento orgânico encontrados em periódicos e eventos Anpad entre os anos de 1997 a 2011.

\subsection{Análise da publicação brasileira sobre o comportamento do consumidor de alimento orgânico entre 1997 a março de 2011}

Considerando-se o período analisado, o estudo apontou que na academia brasileira, todos os artigos que pesquisaram o comportamento do consumidor de alimentos orgânicos foram publicados nos últimos seis anos. A Figura 2 apresenta um gráfico com a quantidade de artigos publicados em cada ano.

REAd | Porto Alegre - Edição 76 - N 3 - setembro/dezembro 2013 - p. 620-645 
Uma análise da produção acadêmica brasileira sobre o comportamento do consumidor de alimento orgânico entre 1997 a 2011

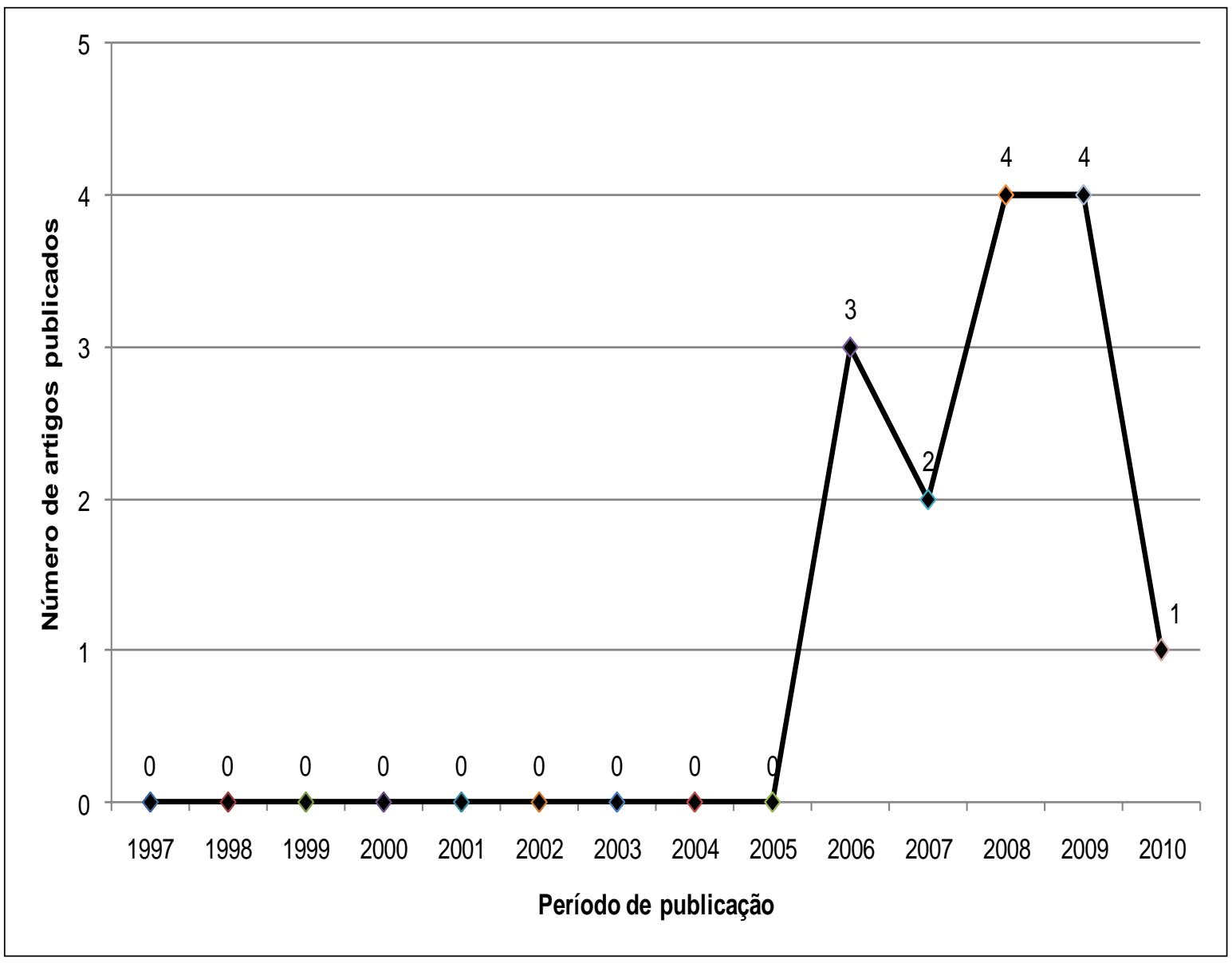

Figura 2 - Gráfico da quantidade de artigos de comportamento do consumidor de alimento orgânico publicado no Brasil entre janeiro do ano de 1997 a março de 2011 Fonte: Elaborada pelos autores, dados da pesquisa

Quanto ao gênero dos autores, considerando-se os 29 autores dos 14 artigos, a pesquisa apontou que 59\% são homens. Quanto à titulação, a maioria é de doutores (69\%), os mestres englobam 24\% dos autores e os demais são pós-doutores.

Com relação ao estado de origem das Instituições de Ensino Superior (IES) dos autores, concluiu-se que $34 \%$ são do Rio Grande do Sul; 24\% são de Minas Gerais; tanto Ceará como Mato Grosso do Sul são o estado de origem de 10\% dos pesquisadores; com 7\% aparecem tanto São Paulo como Paraná; e com 01 artigo publicado, que corresponde a $4 \%$ do total, tem-se Espírito Santo e Paraíba.

Ao verificar as IES por região, a concentração torna-se mais nítida: 41\% estão no Sul e $34 \%$ no Sudeste. A Tabela $1^{\mathrm{i}}$ apresenta as Instituições de Ensino Superior (IES) que os autores estão vinculados. 
Danilo de Oliveira Sampaio, Marlusa Gosling, André Francisco Alcântara Fagundes \& Caissa Veloso e Sousa

Tabela 1 - Distribuição dos autores dos artigos em relação às Instituições de Ensino Superior vinculados

\begin{tabular}{lccc}
\hline \multicolumn{1}{c}{ Instituição } & Quantidade de autores & $\mathbf{\%}$ & Estado \\
\hline UFV - Universidade Federal de Viçosa & 03 & $10,34 \%$ & MG \\
UCDB - Universidade Católica Dom Bosco & 03 & $10,34 \%$ & MS \\
UECE - Universidade Federal do Ceará & 03 & $10,34 \%$ & CE \\
UNISINOS - Universidade do Vale do Rio dos Sinos & 03 & $10,34 \%$ & RS \\
UFRGS - Universidade Federal do Rio Grande do Sul & 03 & $10,34 \%$ & RS \\
UNIJUI - Universidade de Juí & 03 & $10,34 \%$ & RS \\
UFSCAR - Universidade Federal de São Carlos & 02 & $6,90 \%$ & SP \\
UFLA - Universidade Federal de Lavras & 02 & $6,90 \%$ & MG \\
PUC-PR - Pontifícia Universidade Católica do Paraná & 02 & $6,90 \%$ & PR \\
UNIFAL - Universidade Federal de Alfenas & 01 & $3,45 \%$ & MG \\
UFES - Universidade Federal do Espírito Santo & 01 & $3,45 \%$ & ES \\
IFTM - Instituto Federal do Triângulo Mineiro & 01 & $3,45 \%$ & MG \\
UFPB - Universidade Federal da Paraíba & 01 & $3,45 \%$ & PB \\
UERGS - Universidade Estadual do Rio Grande do Sul & 01 & $3,45 \%$ & RS \\
\hline Total de professores & $\mathbf{2 9}$ & $\mathbf{1 0 0 \%}$ & \\
\hline
\end{tabular}

Fonte: Elaborada pelos autores, dados da pesquisa

Ao se considerar o método de pesquisa empreendido nos artigos analisados, foi possível constatar que todos representam estudos empíricos. No tocante à abordagem, 09 trabalhos são qualitativos (64\%); 03 são quantitativos (22\%); e 02 utilizam as duas abordagens (14\%). Quanto à natureza da pesquisa, 05 são classificadas como exploratórias (36\%); o mesmo número é de natureza explicativa (36\%); e 04 artigos são descritivos (28\%). No concernente ao à metodologia de pesquisa em si utilizada nessas pesquisas, a Figura $3^{\text {ii }}$ apresenta os resultados encontrados no estudo.

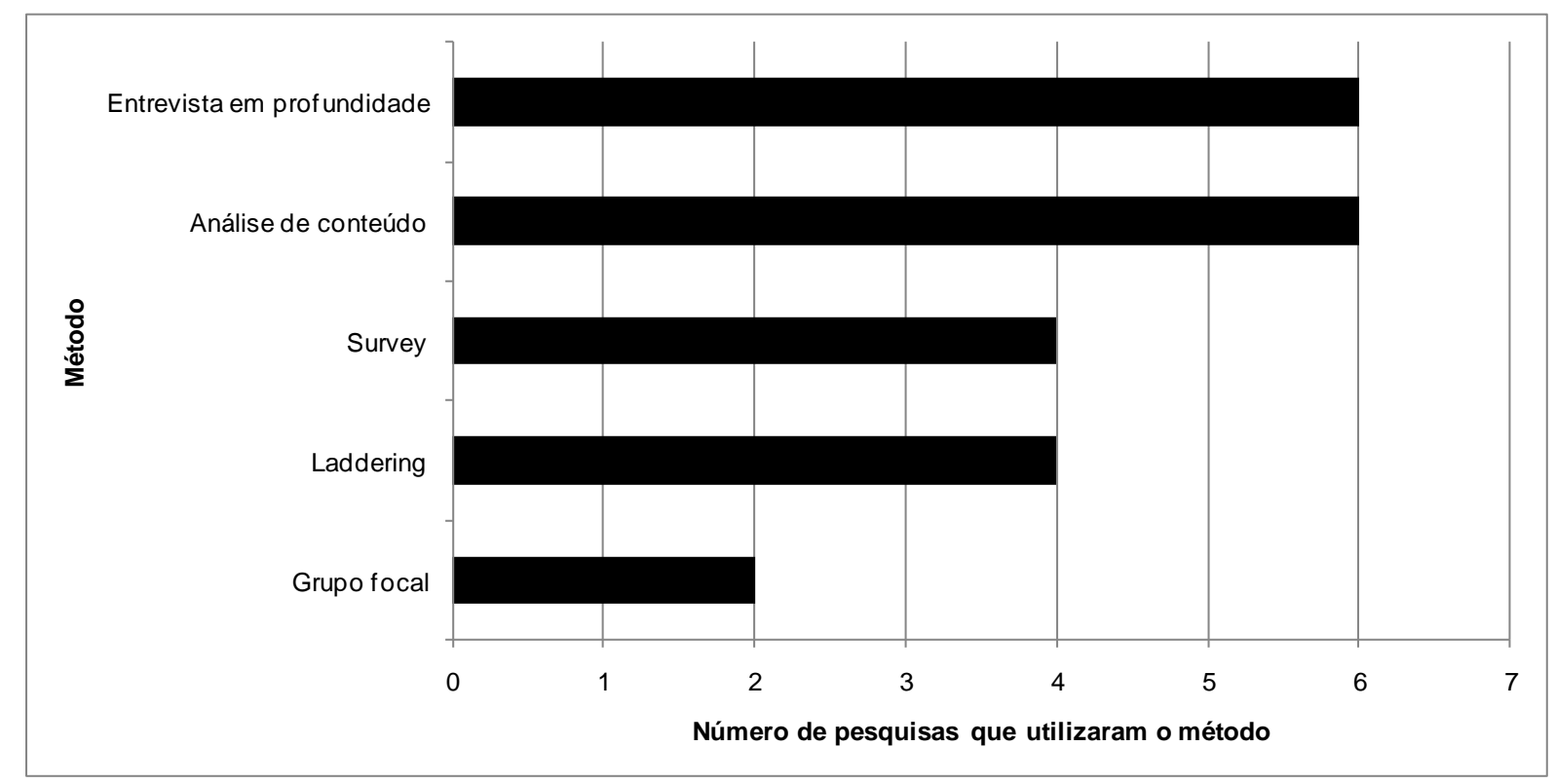

Figura 3 - Metodologias utilizadas pelos autores nos artigos pesquisados REAd | Porto Alegre - Edição 76 - N 3 - setembro/dezembro 2013 - p. 620-645 
Uma análise da produção acadêmica brasileira sobre o comportamento do consumidor de alimento orgânico entre 1997 a 2011

Fonte: Elaborada pelos autores, dados da pesquisa

Em relação ao tipo de estudo, todos os artigos analisados desenvolveram estudos transversais em detrimento do longitudinal. Já ao se considerar os tipos de dados coletados, a maioria - 13 artigos, (93\%) - utilizou de dados secundários, enquanto apenas 01 pesquisas os autores optaram pelo usou de dados secundários, fato que pode ser considerado normal em pesquisas sobre o comportamento do consumidor.

No caso das estratégias de coleta dos dados, o principal instrumento utilizado pelos autores foi a aplicação de questionário/entrevista (12 trabalhos, ou $86 \%$ do total). Apenas 01 trabalho optou por questionário eletrônico, a mesma quantidade para questionários entregues

Notou-se que a maioria dos autores pesquisados prefere enquanto metodologia trabalhar com entrevistas em profundidade e análise de conteúdo, contudo, há uma sinalização principalmente nos congressos de que a metodologia survey está demonstrando interesses dos pesquisadores. Outra constatação sobre pesquisas de comportamento do consumidor de alimento orgânico diz respeito à preferência de coleta de dados primários e ao tipo de artigo empírico.

Além da metodologia e dos instrumentos de coleta de dados, buscou-se analisar a estrutura dos artigos em relação a alguns itens que apontam o conteúdo teórico, metodológico e outros cuidados científicos que os autores tiveram ao finalizar os trabalhos para a submissão. Esses dados são apresentados na Tabela 2.

Tabela 2 - Itens que contam nos artigos sobre conteúdo e metodologia

\begin{tabular}{lcc}
\hline Itens verificados se constam nos Artigos & Frequência & \% \\
\hline Teorias e conceitos base & 14 & $100 \%$ \\
Objetivos da pesquisa & 14 & $100 \%$ \\
Questões de pesquisa & 10 & $72 \%$ \\
Hipóteses ou pressupostos & 05 & $36 \%$ \\
Tipos de dados coletados & 14 & $100 \%$ \\
Forma de coleta de dados & 14 & $100 \%$ \\
Tipo de amostra & 14 & $100 \%$ \\
Limites de estudo & 11 & $79 \%$ \\
Recomendações para pesquisas futuras & 11 & $79 \%$ \\
Recomendações para aplicação prática & 11 & $79 \%$ \\
\hline
\end{tabular}

Fonte: Elaborada pelos autores, dados da pesquisa

Por esse trabalho tratar de uma análise das publicações sobre o comportamento do consumidor de alimento orgânico, dados específicos em relação à teoria aqui utilizada a essa temática foram analisados. Neste sentido, observou-se que os autores entrevistaram o 
Danilo de Oliveira Sampaio, Marlusa Gosling, André Francisco Alcântara Fagundes \& Caissa Veloso e Sousa

consumidor de alimento orgânico em diferentes tipos de varejos, com destaque para as feiraslivres locais (09 artigos, 64\% do total) e supermercados (02 artigos, 14\% do total). Os produtos orgânicos mais pesquisados foram: frutas, legumes e verduras. Outros produtos orgânicos industrializados também foram considerados em alguns estudos, contudo esses não fazem parte do escopo de pesquisa desse trabalho.

No que tange ao tipo de agricultura considerada nos artigos analisados, destaca-se a orgânica (citada em 04 trabalhos, 28\% do total) e a agricultura familiar (03 pesquisas, 22\%). Também foi analisado se os artigos tratavam da temática relacionada à certificação de qualidade dos alimentos orgânicos, constatou-se que 08 pesquisas fizeram menção a essa questão (57\%). Um tema que não foi abordado nos artigos analisados é a relação entre o produtor rural e o empregado rural, que é assunto relativamente recorrente quando se estuda questões referentes ao alimento orgânico. Ademais, também não se encontrou citações relacionadas ao meio ambiente, considerando o impacto dos alimentos orgânicos na sua preservação.

\section{CONSIDERAÇÕES FINAIS}

Este estudo objetivou analisar a produção acadêmica nacional sobre o comportamento do consumidor de alimento orgânico. A pequena produção acadêmica pode ser uma consequência do recente interesse pela sociedade em relação ao alimento orgânico, que apenas nos últimos anos tem sido divulgado com mais ênfase pelos meios de comunicação, bem como pela falta de periódicos e congressos acadêmicos sobre o tema.

Foi possível constatar neste estudo que a maioria das publicações analisadas é oriunda de Instituições de Ensino Superior (IES) localizadas na Região Sul e Sudeste. Este dado sugere a necessidade da academia nacional em incentivar outras regiões a pesquisarem $o$ assunto, podendo, para tanto, incentivar e/ou promover eventos científicos específicos sobre a temática. É importante apontar que o presente trabalho não pretendeu defender ou atacar o alimento orgânico enquanto opções de escolha para uma vida mais saudável, mas sim verificar a produção acadêmica do comportamento do consumidor deste tipo de alimento.

A pesquisa do tipo exploratória é mais frequente nos artigos analisados, contudo, foram também encontrados artigos que utilizaram a pesquisa descritiva e explicativa. Nos trabalhos verificados, os pesquisadores optaram em entrevistar os consumidores em feiraslivres e em supermercados, sendo que as frutas, legumes e verduras são os principais tipos de alimentos considerados nos estudos. Neste caso, uma oportunidade de pesquisa está em REAd | Porto Alegre - Edição 76 - No 3 - setembro/dezembro 2013 - p. 620-645 
Uma análise da produção acadêmica brasileira sobre o comportamento do consumidor de alimento orgânico entre 1997 a 2011

verificar como ocorre o consumo de produtos orgânicos ainda não pesquisados, mas que estão disponíveis aos consumidores nos varejos alimentícios tradicionais. Um caso interessante é o estudo do consumo de produtos orgânicos industrializados e embalados em varejos como hipermercados e lojas especializadas, tendo em vista produtos como pães, leites e derivados, vinhos, barras de cereais, café, massas, geléias entre outros. Assim, as análises não se centralizariam apenas em produtos frescos encontrados nas sessões de hortifrutigranjeiros.

Verificando o conjunto de artigos pesquisados, considerando-se às teorias e conceitos base, há um bom entendimento dos autores pesquisados quanto à importância de fundamentar a base conceitual, bem como há preocupações metodológicas como apoiar os estudos segundo o tipo de amostra e o objetivo de pesquisa. O ponto mais frágil está no pouco uso de formulação de hipóteses e pressupostos. Falta também nas pesquisas já realizadas sobre comportamento de consumidor de alimento orgânico, sugerir recomendações de pesquisas futuras e de aplicação prática.

Conclui-se que a presente pesquisa aponta novos rumos e opções que podem vir a ser seguidos nas pesquisas futuras, tendo em vista um tema que tem cada vez mais ganhado um espaço de destaque na sociedade, e que aparenta ser um importante mercado para um futuro próximo no caso brasileiro.

Enquanto limitação deste trabalho, a pesquisa abordou artigos publicados em eventos científicos exclusivamente da Associação Nacional de Pós-Graduação e Pesquisa em Administração (ANPAD), que apesar de ser uma fonte de primeira linha no país, não é a única que aborda o comportamento do consumidor de alimentos orgânicos. Nesse sentido, outros congressos de expressão nacional ou mesmo locais, podem vir a compor a presente pesquisa em oportunidades futuras.

Por fim, sugerem-se alguns caminhos para aprimorar o presente estudo, como: investigar o motivo da seleção de determinados métodos de pesquisa nos artigos em detrimento de outros; expandir o universo amostral para outros periódicos e eventos científicos para além dos aqui pesquisados; e buscar mais dados e informações junto aos autores dos trabalhos desenvolvidos, principalmente referente às motivações deles em relação ao tema e às expectativas de continuação das pesquisas que eles desenvolveram. Ademais, recomenda-se a realização de um novo estudo no futuro para analisar um período semelhante do que foi aqui considerado, tendo como ponto de partida o ano de 2011, objetivando-se analisar o incremento da pesquisa sobre o comportamento de consumidor de alimentos orgânicos.

REAd | Porto Alegre - Edição 76 - N 3 - setembro/dezembro 2013 - p. 620-645 
Danilo de Oliveira Sampaio, Marlusa Gosling, André Francisco Alcântara Fagundes \& Caissa Veloso e Sousa

\section{REFERÊNCIAS}

AJZEN, I.; FISHBEIN, M. The influence of attitudes on behavior. In: ALBARRACÍN, D.; JOHNSON, B.; ZANNA, M. (Eds). The handbook of attitudes. Mahwah, NJ: Erlbaum, 2005. p. 173-221.

ARCHANJO, L. R.; BRITO, K. F. W.; SAUERBECK, S. Alimentos orgânicos em Curitiba: consume e significado. Revista Caderno de Debates, v. 8, 2001.

ARVOLA, A.; VASSALLO, M.; DEAN, M.; LAMPILA, P.; SABA, A.; LAHTEENMAKI, L.; SHEPHERD, R. Predicting intentions to purchase organic food. The role of affective and moral attitude in the Theory of Planned Behavior. Appetite, v. 50, n. 2/3, p. 443-454, 2008.

BAGOZZI, R. P.; GÜRHAN-CANLI, Z.; PRIESTER, J. R. The social psychology of consumer behavior. Buckingham: Open University Press, 2002.

BELIK, W. Agroindústria e Reestruturação Industrial no Brasil: elementos para uma avaliação. Revista de Economia, v. 18, p. 121-136, 1994.

BLACKWELL, R. D.; MINIARD, P. W.; ENGEL, J. F. Comportamento do consumidor. São Paulo: Pioneira Thomson Learning, 2005.

BOTELHO, D.; MACERA, A. Análise Metateórica de Teses e Dissertações da Área de Marketing Apresentadas na EAESP-FGV (1974-1999). In: XXV EnANPAD. 25. 2001, Campinas-SP. Anais... Campinas-SP: ANPAD, 2001.

CESCHIM, G.; MARCHETTI, R. Z. O comportamento inovador entre consumidores de produtos orgânicos: uma abordagem qualitativa. XXXIII EnANPAD, 09 set. Em: Anais..., São Paulo: ENANPAD, 2009.

CHRYSSOCHOIDIS, G. Repercussions of consumer confusion for late introduced differentiated products. European Journal of Marketing, v. 34, n. 5/6, p. 705-722, 2000.

COLTRO, A. O comportamento do consumidor consciente como fonte de estímulos de mercado às ações institucionais sócio-ambientais. In: I Seminário sobre Sustentabilidade, Centro Universitário FAE, Curitiba, PR, out., 2006.

CONWAY, G. R.; BARBIER, E. B. After the green revolution: sustainable agriculture for development. Earthscan Publications: London, 1990. 
Uma análise da produção acadêmica brasileira sobre o comportamento do consumidor de alimento orgânico entre 1997 a 2011

CRINNION, W. J. Organic foods contain higher levels of certain nutrients, lower levels of pesticides, and may provide health benefits for the consumer. Alternative Medicine Review, v. 15, n. 1, p. 04-12, 2010.

CUPERSCHMID, N. R. M.; TAVARES, M. C. Atitudes em Relação ao Meio Ambiente e sua Influência no Processo de Compra de Alimentos. Revista Interdisciplinar de Marketing (RIMAR), v.1, n.3, p. 05-14, set./dez. 2002.

DEAN, M.; RAATS, M. M.; SHEPHERD, R. Moral concerns and consumer choice of fresh and processed organic foods. Journal of Applied Social Psychology, v. 38, n. 8, p. 20882107, 2008.

ENGEL, J. F.; BLACKWELL, R. D.; MINIARD, P. W. Comportamento do Consumidor. 8. ed. Rio de Janeiro: LTC, 2000.

FAGUNDES, A. F. A.; SAMPAIO, D. de O.; SOUZA, C. V. ; LARA, J. E. Marketing esportivo: um estudo exploratório da publicação acadêmica brasileira. In: IV Encontro de Marketing da ANPAD (EMA). Anais..., Florianópolis: ANPAD, 2010.

FIBL. Research Institute of Organic Agriculture. 2008. Disponível em: <http://www.FIBL.org/en/about-us/activity-report.html>. Acesso em: 17 de mar. de 2010.

FIEP. Federação das Indústrias do Estado do Paraná. Disponível em: $<$ https://rotaagroalimentar.wordpress.com/2010/10/18/classes-c-e-d-sao-as-que-menoscompram-alimentos-organicos/>. Acesso em: 08 de jan. de 2011.

FLICK, U. Uma introdução à pesquisa qualitativa. 2. ed. Porto Alegre: Bookman, 2004.

GOTSCHI, E.; VOGEL, S.; LINDENTHAL, T.; LARCHER, M. The role of knowledge, social norms, and attitudes toward organic products and shopping behavior: survey results from high school students in Vienna. The Journal of Environmental Education, v. 41, n. 2, p. 88-100, 2010.

GUILLON, F.; WILLEQUET, F. Les aliments santé : marché porteur ou bulle marketing ? In : DÉMÉTER. Economie et strategies agricoles: agriculture et alimentation. Paris: Armand Colin, 2003.

GUTHMAN, J. Regulating meaning, appropriating nature: the codification of California organic agriculture. Antipode, v. 30, n. 2, p. 135-154, 1998.

HAWKINS, D.; MONTHERSBAUGH, L.; BEST, R. J. Comportamento do consumidor: construindo a estratégia de marketing. Rio de Janeiro: Elsevier, 2007. 
Danilo de Oliveira Sampaio, Marlusa Gosling, André Francisco Alcântara Fagundes \& Caissa Veloso e Sousa

HOLBROOK, M. B. What is consumer research? Journal of Consumer Research, v. 14, 128-132, jun. 1987.

HOPPE, A.; BARCEllos, M. D.; VIEIRA, L. M.; MATOS, C. A. Comportamento do Consumidor de Produtos Orgânicos: uma aplicação da Teoria do Comportamento Planejado. XXXIV EnANPAD, set. 10. Anais..., Rio de Janeiro: ANPAD, 2010.

KRISCHKE, P. J.; TOMIELLO, N. O comportamento de compra dos consumidores de alimentos orgânicos: um estudo exploratório. Cadernos de Pesquisa Interdisciplinar de Ciências Humanas, v. 10, n. 96, p. 27-43, 2009.

MAPA - Ministério da Agricultura, Pecuária e Abastecimento. Disponível em <http://www.prefiraorganicos.com.br>. Acesso em: 03 de jan. de 2011.

MEDAETS, J. P. P. A construção da qualidade na produção agrícola familiar: sistemas de certificação de produtos orgânicos. Tese (Doutorado em Gestão e Política Ambiental). Universidade de Brasília (UnB). Brasília, 2003.

MENEZES, D. C; PADULA, A. D; CALLEGARO, C. A. M. Internacionalização da oferta de vinho orgânico: um estudo sobre os valores pessoais e motivações de compra entre consumidores internacionais. III Encontro de Estudos em Estratégia. Anais... São Paulo: ANPAD, 2007.

PERIN, M. G.; SAMPAIO, C. H.; FROEMMING, L. M. S.; LUCE, F. B. A Pesquisa survey em artigos de marketing nos Enanpads da década de 90. In: XXIV EnANPAD, 24, Florianópolis. Anais... Florianópolis: ANPAD, 2000.

PETER, J. P.; OLSON, J. C. Comportamento do consumidor e estratégia de marketing. 8. ed. São Paulo: McGraw-Hill, 2009.

PIMENTA, M. L.; VILAS BOAS, L. H. B. Percepção de consumidores de alimentos orgânicos na cidade de Uberlândia na perspectiva de valores: uma aplicação da laddering e cadeia de meios e fins. In: ENCONTRO DE MARKETING DA ANPAD. 3., 2008. Curitiba. Anais... Curitiba: ANPAD. 1 CD-ROM.

PIMENTA, V. P.; SENA, J. O. A.; MOURA, L. P. P; CUNHA, F. A. D.; RUPP, L. M.; HISANO, L. K.; CALDAS, R, G.; FREITAS, I. D.; VIEIRA, D. T. Percepção dos consumidores quanto aos produtos orgânicos na região de Maringá, Paraná, Brasil. Revista Brasileira de Agroecologia, v. 9, n. 2, nov. 2009.

PINTO, M. de R.; LARA, J. E. A Pesquisa na Área do Comportamento do Consumidor: Uma Análise da Produção Acadêmica Brasileira Entre 1997 e 2006. In: XXXI EnANPAD. 31. 2007, Rio de Janeiro-RJ. Anais... Rio de Janeiro-RJ: ANPAD, 2007. 
Uma análise da produção acadêmica brasileira sobre o comportamento do consumidor de alimento orgânico entre 1997 a 2011

RICHARDSON, R. J. Pesquisa Social: Métodos e Técnicas. 3. ed. São Paulo: Atlas, 2007.

ROE, E. J. Things becoming food and the embodied, material practices of an organic food consumer, European Society for Rural Sociology: Blackwell Publishing: Oxford, UK, Sociologia Ruralis, v. 46, n. 2, 2006.

RUCINSKI, J.; BRANDENBURG, A. Consumidores de orgânicos em Curitiba. I Encontro da Associação Nacional de Pós-Graduação em Ambiente e Sociedade, nov. 2008. Anais... Indaiatuba-SP: ANPPAS, 2002.

SHEPHERD, R.; MAGNUSSON, M.; SJÖDÉN, Per-Olow. Determinants of consumer behavior related to organic foods. Royal Swedish Academy of Sciences, v. 34, n. 4-5, jun., 2005 .

SHETH, J. N.; GARDNER, D. M.; GARRETT, D. E. Marketing Theory: Evolution and Evaluation. Chichester: John Wiley \& Sons, 1988.

SLUZZS, T.; PADILHA, A. C. M.; MATTOS, P. Inovações em organizações do agronegócio: análise em uma organização produtora de chá orgânico. XXV Simpósio de Gestão da Inovação Tecnológica. Anais... Brasília: ANPAD, 2008.

SOLOMON, M. R. O comportamento do consumidor: comprando, possuindo e sendo. 5 . ed. Porto Alegre: Bookman, 2002.

SOUZA, M. C. M. Cafés sustentáveis e denominações de origem: a certificação de qualidade na diferenciação de cafés orgânicos, sombreados e solidários. Tese (Doutorado em Ciência Ambiental). Universidade de São Paulo (USP), São Paulo, 2006.

STRINGHETA, P. C.; MUNIZ, J. R. Alimentos orgânicos: produção, tecnologia e certificação. UFV: Viçosa, 2003.

TARKIAINEN, A.; SUNDQVIST, S. Subjective norms, attitudes and intentions of Finnish consumers in buying organic food. British Food Journal, v. 107, n. 11, p. 808-822, 2005.

VIEIRA, F. G. D. Panorama acadêmico-científico e temáticas de estudos de marketing no Brasil. In: XXIV EnANPAD, 24, Florianópolis. Anais... Florianópolis: ANPAD, 2001.

VILAS BOAS, L. H. B.; BRITO, M. J.; SETTE, R. S. Perspectivas da Antropologia no Estudo do Comportamento do Consumidor: contribuições para o desenvolvimento da teoria e pesquisa de marketing. Revista Eletrônica de Administração, v. 12, n. 2, mar/abr 2006. 
Danilo de Oliveira Sampaio, Marlusa Gosling, André Francisco Alcântara Fagundes \& Caissa Veloso e Sousa

VILAS BOAS, L. H. B; SETTE, R. S.; BRITO, M. J. de. Comportamento do consumidor de produtos orgânicos: uma aplicação da teoria da cadeia de meios e fins. Organizações Rurais \& Agroindustriais, v. 8, n. 1, p. 25-39, 2006.

VILAS BOAS, L. H. B; SETTE, R. S.; PIMENTA, M. L. Comportamento do consumidor de alimentos orgânicos na cidade de Uberlândia: uma aplicação da técnica laddering. XLVI Congresso da Sociedade Brasileira de Economia, Administração e Sociologia Rural, set. 09. Anais... Londrina: SOBER, 2008.

WILLIAMSON, C. S. Is organic food better for our health? British Nutrition Foundation, $\mathrm{n}$. 32, p. 104-108, 2007.

ZAMBERLAN, L.; BÜTTENBENDER, P. L.; SPAREMBERGER, A. XXX EnANPAD, 30, set. 06. Anais... Salvador-BA: ANPAD, 2002.

ZANOLI, R.; NASPETTI, S. Consumer motivations in the purchase of organic food: a means-end approach. In: LXXII EAAE, Organic Food Marketing Trends. In: Seminar, Chania, Greece, 2001.

ZUIN, L. F. S.; QUEIROZ, T. R. Agronegócios: gestão e inovação. São Paulo: Saraiva, 2006.

\footnotetext{
${ }^{\mathrm{i}}$ As Instituições de Ensino as quais os pesquisadores são ligados foram verificadas por meio da Plataforma Lattes, no Currículo de cada pesquisador.

${ }^{\text {ii }}$ Alguns autores usaram mais de uma metodologia em seus estudos.
} 